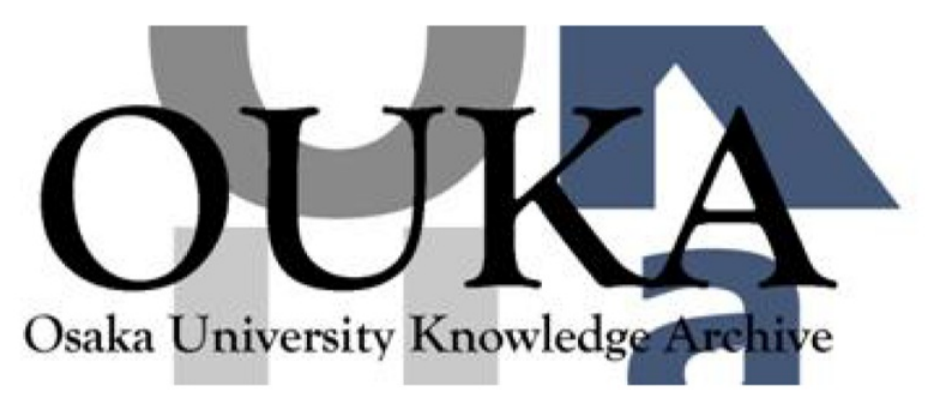

\begin{tabular}{|c|l|}
\hline Title & $\begin{array}{l}\text { New technique for the observation of x-ray CTR } \\
\text { scattering by using an imaging plate detector }\end{array}$ \\
\hline Author(s) & Shimura, Takayoshi; Harada, Jimpei \\
\hline Citation & $\begin{array}{l}\text { Journal of Applied Crystallography. 26(2) } \\
\text { p. 151-p. 158 }\end{array}$ \\
\hline Issue Date & $1993-04-01$ \\
\hline oaire:version & VoR \\
\hline URL & https://hdl. handle. net/11094/85529 \\
\hline rights & 0 International Union of Crystallography \\
\hline Note & \\
\hline
\end{tabular}

Osaka University Knowledge Archive : OUKA

https://ir. Library. osaka-u. ac. jp/

Osaka University 


\title{
A New Technique for the Observation of X-ray CTR Scattering by Using an Imaging Plate Detector
}

\author{
By Takayoshu ShImlra and Jimpei Harada
}

Department of Applied Physics, Nagoya University, Chikusa-ku, Nagoya 464-01, Japan

(Received 17 August 1992; accepted 29 September 1992)

\begin{abstract}
A new technique, using an Imaging Plate (IP) detector, was developed for the observation of X-ray crystal truncation rod (CTR) scattering. The use of an IP detector in conjunction with a synchrotron-radiation (SR) source is very effective for the observation of CTR scattering. The advantages and disadvantages of this technique are indicated by examples of the observation of CTR scattering from various samples: a naturally oxidized surface of an $\mathrm{Si}(111)$ wafer; $\mathrm{MBE}$-grown GaAs/AlAs/GaAs on $\mathrm{GaAs}(001)$ substrate; Al-capped GaAs on an $\mathrm{Si}(111)$ substrate; and a cleavage $\mathrm{NaCl}(001)$ surface. It is also shown that it is possible to convert the observed intensity of the CTR scattering to an absolute scale if a stationary photograph is taken.
\end{abstract}

\section{Introduction}

The Fuji Photo Film Co. has developed a new type of $\mathrm{X}$-ray area detector, referred to as an Imaging Plate (IP), and read-out system (Miyahara, Takahashi, Amemiya, Kamiya \& Satow 1986). The IP is a flexible plastic plate coated with fine photostimulable phosphor crystals, $\mathrm{BaFBr}: \mathrm{Eu}^{2+}$. The X-ray image is stored on the phosphor screen as a distribution of quasistable color centers. The stored image can be read by measuring the intensity of fluorescence which is stimulated by scanning the surface of the phosphor screen with a focused He-Ne laser beam. The IP detector has the advantages of high detective quantum efficiency, wide dynamic range of $1-10^{5}$, spatial resolution of $\sim 100 \mu \mathrm{m}$, approximately $20-60$ times higher sensitivity than that of high-sensitivity X-ray film, large activity area of $400 \times 200 \mathrm{~mm}$ and unlimited counting rate. This system, therefore, has been used for a variety of $\mathrm{X}$-ray studies; for example, powder diffraction from crystals in a diamond-anvil cell and small-angle X-ray scattering from muscle and a protein crystal (Amemiya, Matsushita, Nakagawa, Satow, Miyahara \& Chikawa 1988). It is also recognized as a remarkably effective system, especially in studies of protein crystals (Sakabe 1991).

$\mathrm{X}$-ray diffraction studies of crystal surfaces and interfaces have been an active field for several years with the

$0021-8898 / 93 / 020151-08 \$ 06.00$ appearance of synchrotron-radiation sources because of the non-destructive nature and low interaction with materials. When we measure the X-ray scattering from a truncated crystal surface, a rod-shaped scattering is observed emanating along the surface normal direction from each Bragg point. This is a diffraction effect due to abrupt truncation of the periodicity of the lattice at the surface (Andrews \& Cowley, 1985) and is referred to as crystal truncation rod (CTR) scattering (Robinson, 1986). The distribution of the intensity along the CTR scattering is very sensitive to the morphology of the crystal surface on an atomic scale. The analysis of such CTR scattering provides diverse information about the crystal surface (see, for example, Harada \& Kashiwagura, 1989). But the observation of the CTR scattering is not easy because it is a needle shape in reciprocal space rather than a rod, the width of which is, in most cases, as narrow as the Bragg point (Kashiwagura, Kashihara, Sakata, Harada, Wilkins \& Stevenson, 1987) and its intensity covers a very wide range, about $1-10^{7}$ (Stevenson, Wilkins, Harada, Kashiwagura, Oshima \& Sakata, 1988). Among our early examinations of all the available techniques to observe the CTR scattering with high precision, one of the most efficient techniques was found to be simple oscillation photography with an IP detector in combination with a synchrotron-radiation source (Harada \& Kashiwagura, 1989; Barnea et al., 1989).

In this paper, we discuss the effectiveness of using the IP detector in conjunction with a SR source for the observation of CTR scattering. The instrumentation and the procedure for this technique are given. The advantages and disadvantages of the technique are discussed with several examples of the observations of CTR scattering.

\section{Instrumentation}

The system developed by Sakabe (1991) for protein crystallography was used for the present study. The schematic arrangement of the system, installed at BL-6 $\mathrm{A}_{2}$ of the Photon Factory, KEK, is shown in Fig. 1. It consists of a bent mirror, a bent Si(111) monochromator and a Weissenberg camera, which can also be used for oscillation-

(C) 1993 International Union of Crystallography 
camera work. A vertically bent mirror of fused quartz is set before the monochromator to increase the brightness and to remove higher harmonics of the incident beam. The monochromator is an asymmetrically cut $\mathrm{Si}(111)$ crystal of triangular shape which is horizontally bent. Four film cassettes of radius $143.25,286.5,430.0$ and $573.0 \mathrm{~mm}$ are prepared. The inside of the cassette can be filled with helium gas in order to reduce the background by air scattering. Several collimators of different sizes, $0.2 \times 0.2$, $0.1 \times 0.1 \mathrm{~mm}$, can also be used. The Fuji BA100 system is employed for the read-out of image data from an exposed IP, where the pixel size of the read-out data is 0.1 $\times 0.1 \mathrm{~mm}$ and the intensity is digitized to 1024 levels in the range $1-10^{4}$. The IP can be used many times if the stored image is erased after each read-out.

In the present work, the size of the collimator slit just before the samples and the selected wavelength are $0.2 \times 0.2 \mathrm{~mm}$ and $1.04 \AA$ for $\mathrm{Si}(111), \mathrm{MBE}$-grown $\mathrm{GaAs}$ on $\mathrm{Si}(111)$ and cleavage $\mathrm{NaCl}(001)$ and $0.1 \times 0.1 \mathrm{~mm}$ and $1.542 \AA$ for MBE-grown $\mathrm{GaAs} / \mathrm{AlAs} / \mathrm{GaAs}$ on $\mathrm{GaAs}(001)$, respectively. We selected the $430.0 \mathrm{~mm}$ cassette for all the samples studied. The angular resolution, therefore, should be about $0.026^{\circ}$ for the $0.2 \times 0.2 \mathrm{~mm}$ collimator slit. The actual angular resolution is poorer because the incident beam is not ideally parallel. It can be estimated to be about $0.066^{\circ}$ (horizontal) and $0.040^{\circ}$ (vertical) by measuring the direct-beam size at the position of the IP.

\section{Observations}

\section{3-1. Oscillation photographs}

A diffraction photograph can be taken by oscillating a sample around the Bragg condition, the well known oscillation photograph. The schematic arrangement of this oscillation mode in reciprocal space is shown in Fig. 2. The figure represents the simplest case for the observation of the CTR scattering, where it elongates from the Bragg point for a lattice plane which is parallel to the surface. The CTR scattering on the higher-order layer line can also be measured if the sample is tilted in such a way that the CTR scattering comes into the region of the equatorial plane.

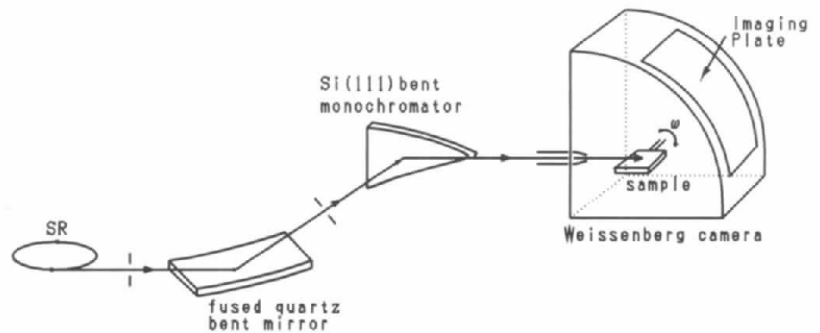

Fig. 1. Experimental arrangement for the observation of X-ray CTR scattering by using an Imaging Plate detector in conjunction with synchrotron radiation. (a) $\mathrm{Si}(111)$. As a typical example of the observation of CTR scattering by using an IP, the result obtained from an $\mathrm{Si}(111)$ single crystal is shown, where the surface was mechanochemically polished by an ordinary wafermaking process. The surface we observed is, therefore, not such a clean surface as is realized in UHV, but is covered with a native oxide layer. A photograph was taken by oscillating the sample around the 111 Bragg point. The oscillation angle $\Delta \omega$ is $\pm 4.9^{\circ}$. The exposure time was $1 \mathrm{~h}$. Readouts of the IP were performed twice for one exposure in order to read different levels of the CTR intensities. It was confirmed that the intensity distribution obtained by the second reading was not influenced beyond the error level by the first reading except for a scale factor. Oscillation photographs for the first and second readings are shown in Fig. 3 and its inset, respectively. The 111 Bragg points are indexed in the photograph. The intensity representation is given on a logarithmic scale.

Elliptically shaped diffuse scattering seen around the Bragg point is the well known thermal diffuse scattering

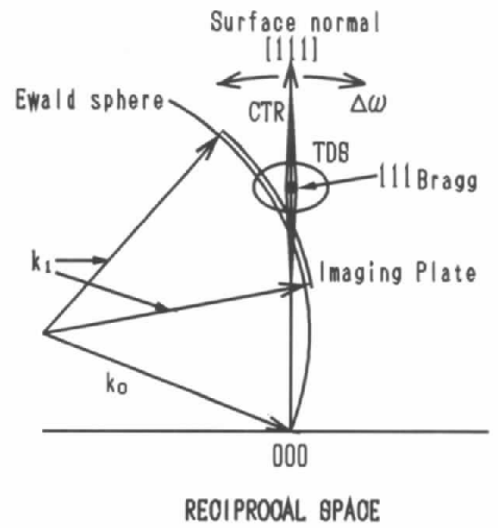

Fig. 2. Schematic arrangement of the oscillation photograph in reciprocal space.

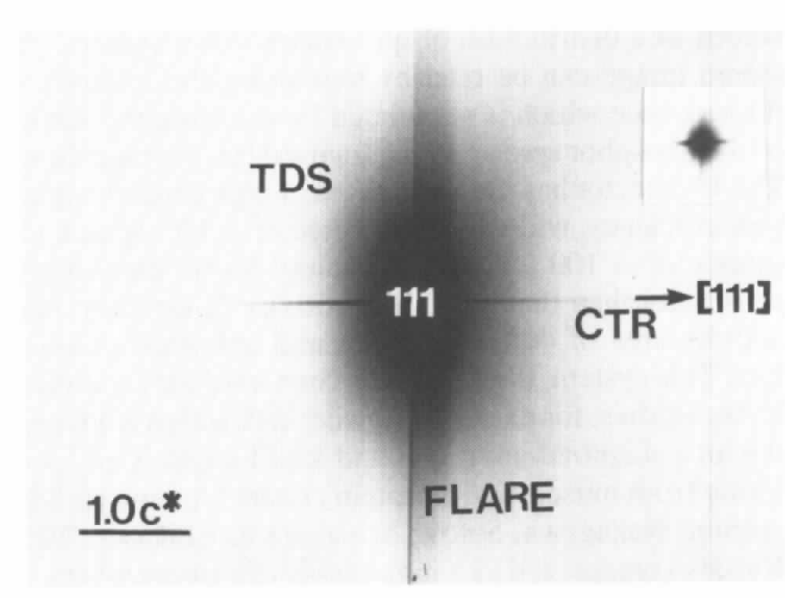

Fig. 3. The oscillation photograph of the CTR scattering with respect to the 111 Bragg point from an $\mathrm{Si}(111)$ wafer. The inset shows the second reading. 
(TDS) and the rod-shaped scattering emanating from the Bragg point along the [111] direction is the CTR scattering. The sharp intensity contrast seen perpendicular to the [111] direction through the Bragg point, marked as FLARE in the photograph, arises during the process of developing a very strong Bragg point on the IP by the Fuji BA 100 system. This is independent of the $X$-ray scattering from a sample. A careful investigation showed that such a flare does not affect the estimation of the intensity distribution of the CTR scattering if the transfer direction, perpendicular to the scanning direction, is parallel to the CTR scattering as shown in the Appendix, because it is subtracted as background. The intensities, which were integrated over five pixels along the CTR scattering, were plotted along the line perpendicular to the CTR scattering. One of the profiles is shown in Fig. 4. The majority of the background comes from TDS and can easily be subtracted. The intensities of the CTR scattering can easily be integrated. The intensity distribution of the CTR scattering thus obtained is shown in Fig. $5(a)$ for the first and second readings and in Fig. $5(b)$ for the normalized intensity distributions. The solid line in Fig. $5(b)$ shows the calculated curve for an ideally sharp truncated surface. The observed intensities deviate from the calculation, especially at the foot of the Bragg point, indicating that the interface between the Si surface and the oxidized layer has some characteristic structure (Kashihara, Kawamura, Kashiwagura \& Harada 1987).

(b) GaAs/AlAs/GaAs on GaAs(001). This sample has the structure of GaAs/AlAs/GaAs grown on a GaAs(001) substrate by the MBE method. The nominal numbers of molecular layers of GaAs, AlAs and GaAs are 1240, 125 and 1240, respectively. Kashihara, Ikeda \& Harada (1989) have examined the CTR scattering around the $002 \mathrm{Bragg}$ point from this sample by using a 4-circle diffractometer with synchrotron radiation at beam line $4 \mathrm{C}$ of the Photon Factory, KEK. The measurement was performed by the $\theta-2 \theta$ scanning mode. The observed intensity profile showed a Laue-function-like modulation owing to interference between the three CTR scatterings from the top surface, the interface between $\mathrm{GaAs}$ and $\mathrm{AlAs}$ and that

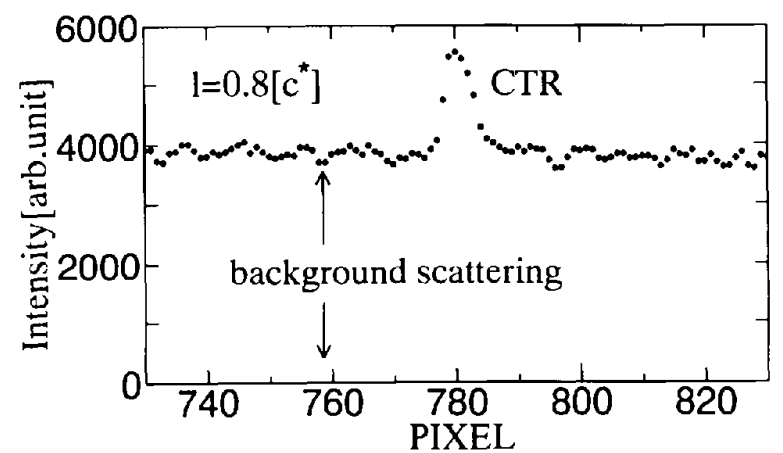

Fig. 4. The intensity profile along the line perpendicular to the CTR scattering for $l=0.8 c^{*}$, as a typical example. between AlAs and GaAs. As assigned by the previous authors, the oscillation of large period is due to 125 molecular layers of AlAs and the faint oscillation with short period is due to 1240 layers of GaAs on AlAs. From the analysis of the periodicity of the oscillation of the intensity, the lattice spacing and the number of molecular layers of $\mathrm{GaAs}$ and AlAs were well estimated. But it was difficult to obtain further information about surface and interface roughnesses by analyzing the intensity data obtained by the $\theta-2 \theta$ scanning mode because the background scatterings were not properly subtracted.

We therefore re-examined this sample by the present technique using the IP detector. The oscillation photograph of the CTR scattering taken around the 002 Bragg point is shown in Fig. 6. We can see the array of peaks along the [001] direction through the 002 Bragg point, indicating that the CTR scattering is not a simple rod but has a periodic modulation along the rod. The intensity integrated across the rod can easily be obtained in the way

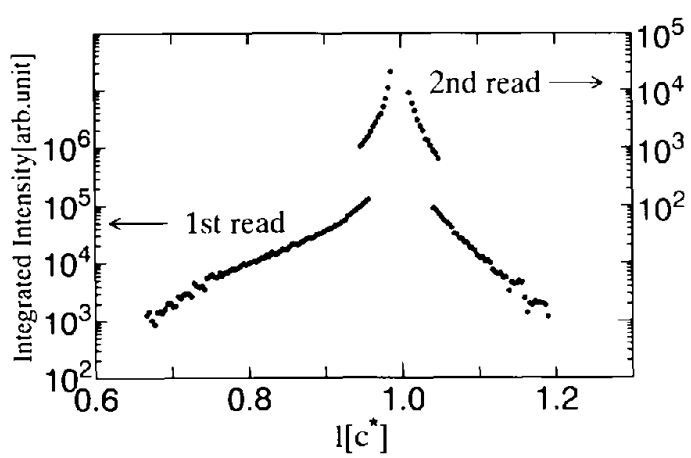

(a)

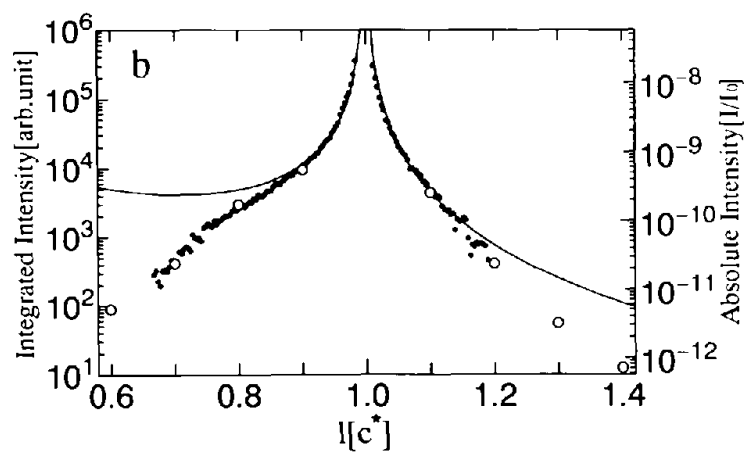

(b)

Fig. 5. Intensity distributions along the CTR scattering from an Si(111) wafer; $(a)$ for the first and second readings, $(b)$ for the normalized intensity distribution. In $(b)$, the solid curve shows the calculation for an ideally sharp truncated surface and the open circles show the results obtained by the stationary-photograph mode. The absolute scale is given on the right-hand axis, which represents the ratio of the scattered intensity to the incident beam. It should be noted that CTR scat tering is of the order of $10^{-12}$ of the incident-beam intensity. 
mentioned in $\S 3-1(a)$. Thus, we can subtract the background properly. Fig. 7 shows the results, where the dots indicate the observed integrated intensities.

Comparing the present result with that obtained by the $\theta-2 \theta$ scanning mode using the 4-circle diffractometer, we see that the faint oscillation with fine period cannot be resolved in the present observation while it is clearly seen in the previous data. This is due to poor resolution along the CTR scattering in the present observation. But the quality of the relative intensities was much higher than the previous data so that the least-squares-fitting analysis can be applied. The solid curve in the figure represents the calculation based on a model obtained by the least-squares fitting. We see a good agreement between the observation and a model calculation. It was confirmed from analysis that the roughness of the interface between $\mathrm{GaAs}$ and AlAs and also that between AlAs and GaAs could be obtained by the least-squares fitting together with the lattice spacing and the number of molecular layers of AlAs.

\section{CTR}

$$
002
$$

\section{$\underline{0.05} \mathrm{a}^{*}$}

Fig. 6. The oscillation photograph around the 002 Bragg point for a MBE-grown GaAs/AlAs/GaAs epitaxic-layer system on a $\mathrm{GaAs}(001)$ substrate. The periodic array of dots is due to the interference effect of two interface boundaries $\mathrm{GaAs} / \mathrm{AlAs}$ and $\mathrm{AlAs} / \mathrm{GaAs}$.

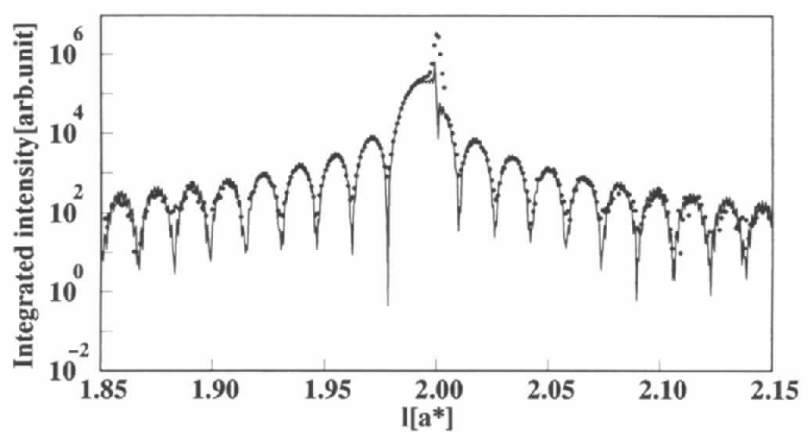

Fig. 7. The intensity distribution along the CTR scattering around the 002 Bragg point obtained from Fig. 6 . The solid curve indicates the model calculation where the lattice spacings and the interface roughnesses are adjusted. (c) Al-capped GaAs on $\mathrm{Si}(111)$. A hetero-epitaxic sample of $\mathrm{GaAs}$ on an $\mathrm{Si}(111)$ substrate grown by the MBE method was investigated. The nominal number of molecular layers of GaAs is 4 in this case. In order to protect the $\mathrm{GaAs}$ thin layers from oxidization, aluminium is capped as a buffer layer, with thickness about $1000 \AA$. The oscillation photograph around the 111 Bragg point of the $\mathrm{Si}(111)$ substrate is shown in Fig. 8. The 111 Bragg point is indexed. We see that the CTR scattering is very asymmetric with respect to the Bragg point. The CTR scattering on the higher-angle side decreases very rapidly with the distance from the Bragg point. This is understood to be due to the existence of the GaAs epitaxic layer on the Si substrate, since the CTR scattering on the higher-angle side from the $\mathrm{Si}(111)$ wafer should be strong as seen in Fig. 3. We see a Debye-Scherrer ring-like scattering in the photograph, which is identified to be the 111 Bragg reflection from the aluminium layer. From the intensity distribution along the ring, we see that aluminium in the cap layer also has a preferred orientation along the [111] direction. It is one of the great advantages of X-ray scattering that the CTR scattering from the interface between GaAs and the Si substrate can be observed separately from the aluminium scattering.

The intensities integrated across the rod can easily be obtained in the same way as indicated in $\S 3-1(a)$. The integrated intensity distribution along the CTR is shown in Fig. 9. The dots and the solid line indicate the observed intensity and a model calculation, respectively. If the calculation is fitted to the observed intensity data, several parameters, such as the roughness of the interface of the GaAs layer and Si substrate and the interfacial separation, can be adjusted. The results obtained by this least-squares fitting will be given in a separate paper together with a discussion on the interface structure on an atomic scale (Shimura, Harada \& Kawamura, 1993).

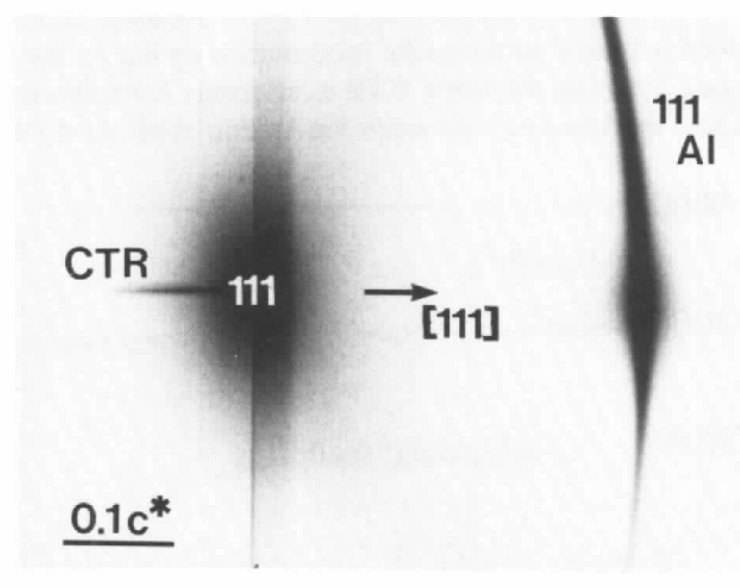

Fig. 8. The oscillation photograph around the 111 Bragg point for the Al-capped GaAs on an $\mathrm{Si}(111)$ substrate. A Debye-Scherrer ring-like scattering is observed which is identified as the 111 Bragg reflection from the aluminium layer. 


\section{3-2. Stationary photographs}

(a) $\mathrm{Si}(111)$. In general, it is possible to take any diffraction photograph by keeping a sample stationary near the Bragg condition. The intensity distribution obtained by this mode represents the section of the Ewald sphere in reciprocal space. As far as the CTR scattering is concerned, the cross section of the CTR scattering with the Ewald sphere is a simple dot on the IP. However, TDS around the Bragg point is also observed as a diffuse peak.

In this examination we used the same sample as that used in $\S 3-1(a)$. One of the stationary photographs is shown in Fig. 10. We see a simple dot as well as a broad diffuse peak, as expected. In this photograph, the flare is not noticed because no spot is strong enough to create the flare. The intensity distribution along the line between the

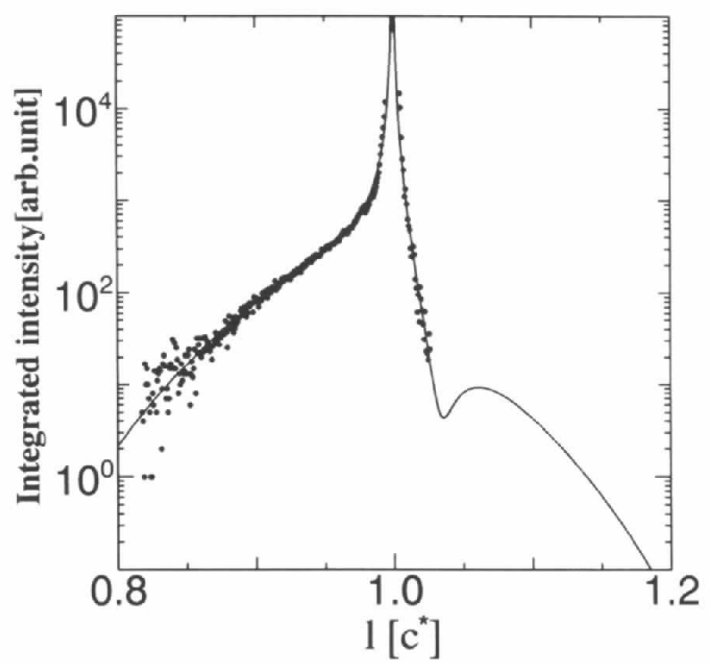

Fig. 9. The intensity distribution of the CTR scattering around the 111 Bragg point for the Al-capped $\mathrm{GaAs}$ on an $\mathrm{Si}(111)$ substrate. The solid curve indicates the model calculation in which the roughness of the interface of the GaAs layer and Si substrate and the interfacial separation are adjusted.

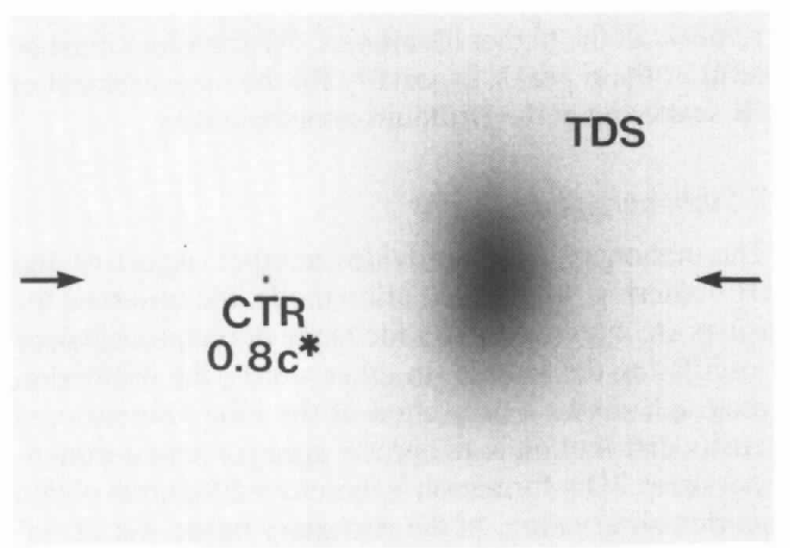

Fig. 10. The stationary photograph taken near the 111 Bragg point for an $\mathrm{Si}(111)$ wafer. The sharp spot is the section of the CTR scattering at $l=0.8 c^{*}$ and the broad scattering is TDS. arrows is plotted in Fig. 11(a). In this figure, the angle of the sample is set to $-1.92^{\circ}$ from the 111 Bragg point $\left(l=0.8 c^{*}\right)$. We see a sharp spot and a diffuse scattering which are identified as the sections of the CTR scattering and TDS with the Ewald sphere near the 111 Bragg point. The intensity of the TDS can easily be calculated if the elastic constants and absorption coefficient of Si are known (Wooster, 1962). In Fig. 11(b), we show how calculated TDS fits with observation, two parameters, such as incident beam and background level, being adjusted. By this fitting we can also obtain the intensity of the CTR scattering at $l=0.8 c^{*}$ on an absolute scale by simply comparing the integrated intensity of the sharp scattering with TDS.

It should also be noted that the signal-to-background ratio of the CTR scattering is much better in the stationary photograph (Fig. 11a) than in the oscillation photograph (Fig. 4). It is therefore possible to obtain even very weak CTR scattering around the Brillouin-zone boundary in this way. In Fig. 5(b), open circles show the results obtained by the stationary-photograph method. They are plotted with the data (solid circles) obtained from the oscillation photograph for comparison. As shown in Fig. $5(b)$, the agreement between the two observations is extremely good. In addition, the absolute scale is given on the right-hand axis.

(b) $\mathrm{NaCl}(001)$. According to the study by Kashihara, Kimura \& Harada (1989), our $\mathrm{NaCl}$ crystal has a mosaic structure. We have carried out a study to see the effect of

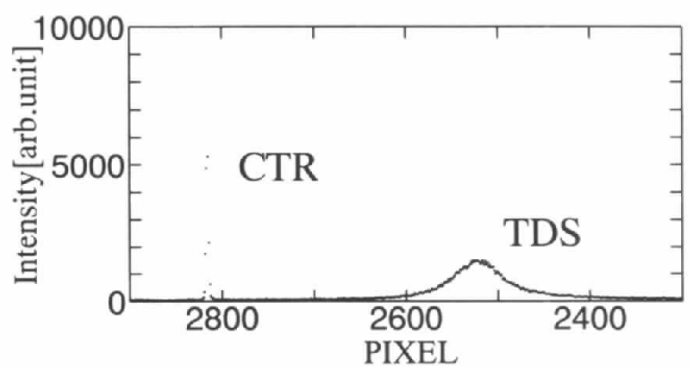

(a)

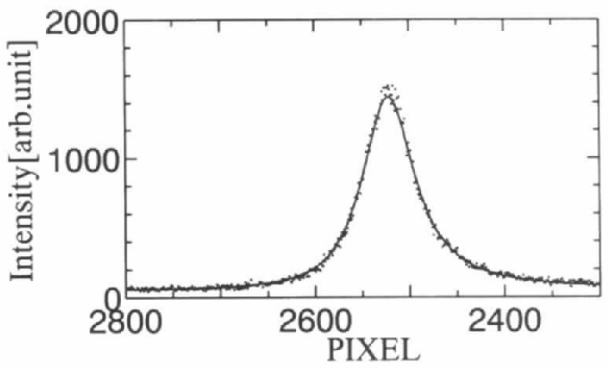

(b)

Fig. 11. (a) The intensity distribution along the line between the arrows in Fig. 10. The sharp peak is the CTR scattering at $l=0.8 c^{*}$ and the broad peak is TDS. (b) The result obtained by least-squares fitting for TDS, where two parameters, the incident-beam intensity and background level, are adjusted. 
the mosaicity by stationary photograph. The $\mathrm{NaCl}$ sample was cleaved in vacuum and kept in a vacuum capsule. One of the stationary photographs obtained from the cleavage $\mathrm{NaCl}(001)$ surface is shown in Fig. 12. This photograph was taken near the 002 Bragg point of $\mathrm{NaCl}$. Comparing this photograph with that obtained from $\mathrm{Si}$ (Fig. 10), we see that the CTR scattering from the $\mathrm{NaCl}$ consists of several peaks, although the resolution is the same for both cases. We are certain that such a splitting of the CTR scattering indicates the existence of crystal mosaicity. The three-dimensional nature of the mosaicity of the crystal is revealed.

\section{Discussion}

\section{4-1. Oscillation photographs}

As shown by the present study, the IP detector has several advantages as a two-dimensional detector. For one thing, a simultaneous measurement is possible. When an ordinary detector, such as a scintillation counter, is employed, measurements along the line perpendicular to the CTR scattering have to be made, in order to obtain the integrated intensity for each point. In such a measurement, the intensity of the incident beam must be monitored, particularly if a synchrotron-radiation source is employed because its intensity gradually decreases with time. On the other hand, the oscillation-photograph mode does not require the incident-beam monitor because all of the CTR scattering can be observed in one exposure. Thus we can easily obtain relative integrated intensities from one oscillation photograph. Furthermore, two or three CTR scatterings elongated from different Bragg points can be simultaneously measured if the tilt angle of the sample and the angle range of the rotation are selected appropriately. The simultaneity is also very useful if the temperature and the pressure of a sample are controlled. Not only CTR scattering but also thermal diffuse scattering (TDS), the halo

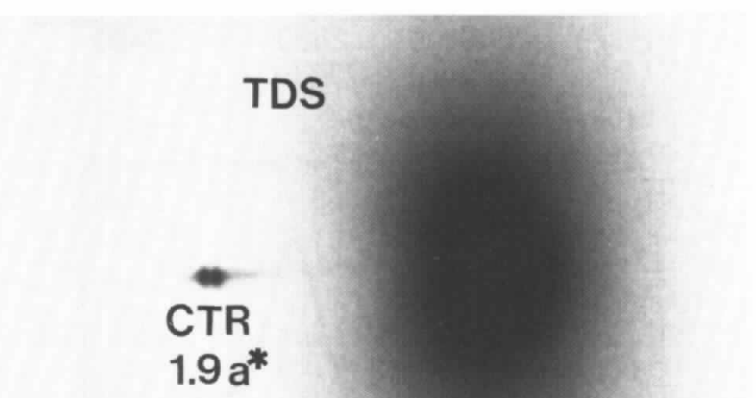

Fig. 12. The stationary photograph taken near the 002 Bragg point for a cleaved $\mathrm{NaCl}(001)$ surface. The sharp spot is the section of the CTR scattering at $l=1.9 a^{*}$ and the broad scattering is TDS. The CTR scattering is observed to be split because of the crystal mosaicity. pattern from amorphous materials, powder-like patterns etc. can be observed. It is, therefore, possible to judge the quality of data as a whole as well as the quality of the crystal from the distribution of these scatterings in a wide area in reciprocal space.

Another advantage of using the IP detector is that the CTR scattering can be observed as a continuous intensity distribution along a line; i.e. continuity of the position of the observed data. The distribution is usually modified in a complicated way, especially for samples with epitaxic layers on the crystal surface. The IP detector is particularly effective when it is hard to deduce what sort of intensity distribution is obtained from a sample.

According to Kashiwagura, Kashihara, Sakata, Harada, Wilkins \& Stevenson (1987), three streaks are observed to be elongated from the Bragg point when a three-crystal arrangement is employed in order to improve the resolution. One of the streaks is the CTR scattering from the sample; the other streaks are the tails of the monochromator (TM) and the analyzer crystal (TA). In the present method, the TA is not a part of the observed intensity since no analyzer crystal is used. The TM, however, has an influence on the observed intensity, particularly the CTR intensity in the vicinity of the Bragg points, because the TM lies on the CTR scattering in the oscillation photograph. We therefore must estimate the intensity of the TM, which depends on the morphology of the surface of the monochromator crystal. If the intensity of the TM is strong, a peak appears between the TDS and the CTR scattering on the stationary photograph taken near the Bragg condition. In this study, the intensity of the TM was weak enough to be ignored because the peak of the TM did not appear on the stationary photograph.

We found a lot of sharp peaks in the oscillation photographs with a very low intensity level. From the positions of the peaks, they were identified as Bragg reflections due to higher harmonics. The mirror is set before the monochromator in the experimental arrangement in order to remove higher harmonics of the incident beam. From the above observation, it is deduced that it is very hard to remove all the higher harmonics. We therefore must be careful of these peaks, especially for the measurement of CTR scattering at the Brillouin-zone boundary.

\section{4-2. Stationary photographs}

The stationary mode provides another aspect of the CTR scattering. In the oscillation mode, the observed intensities are integrated in a wide range of reciprocal space by oscillating the sample. In other words, the oscillation photograph shows a projection of the three-dimensional intensity distribution in reciprocal space on a two-dimensional sheet. 3D information is therefore difficult to obtain from this observation. In the stationary mode, the $2 \mathrm{D}$ intensity distribution in reciprocal space can be projected as $2 \mathrm{D}$ data. As shown by the section of the CTR scattering, valuable information is included about the mosaicity 
of a crystal surface. Furthermore, the macroscopic distribution of the surface morphology, like the roughness averaged in a coherent area of the incident beam, can be obtained from the intensity distribution of a section of the CTR scattering if a wide incident beam with a high degree of parallelism is used.

In the stationary photograph, the $S / N$ ratio is very good compared with the oscillation photograph although the whole of the CTR scattering cannot be observed simultaneously. The CTR scattering around the Brillouin-zone boundary, for which the intensity is usually very weak, can be measured by using this technique as shown in $\S 3-2(a)$ but monitoring of the incident-beam intensity is required in this case.

The various advantages and disadvantages of an ordinary $2 \mathrm{D}$ detector are also applicable to the stationary photograph. One of the great advantages is to be able to obtain the intensity of the CTR scattering on an absolute scale by simply comparing the intensity of the CTR scattering with that of TDS observed simultaneously as shown in $\S 3-2(a)$.

We thank Professor N. Sakabe of the Photon Factory, $\mathrm{KEK}$, for his encouragement to use his camera. We also thank Dr A. Nakagawa for his considerable assistance during the observations, Professor H. Terauchi of KanseiGakuin University for providing us with the sample of MBE-grown GaAs/AlAs/GaAs on GaAs and Mr Kawamura of NTT Applied Electronics Laboratories for the Al-capped $\mathrm{GaAs}$ on $\mathrm{Si}$ sample. This work was supported by the PF, KEK, under proposal no. 90-086 and partly by a Grant in aid for Scientific Research on Priority Areas nos. 030243105 and 04227105 from the Ministry of Education, Science and Culture, Japan. The computer calculations were carried out on the Facom-M780 computer of the Nagoya University Computation Center.

\section{APPENDIX}

The influence of flare on the profile of CTR scattering

Flare arises in the process of developing a point of very strong intensity on an imaging plate by the Fuji BA100 system. It is understood that the flare arises when a weak stray laser light from the focused beam happens to stimulate fluorescent light from the point of a concentrated color center such as a Bragg point. Its appearance depends on the geometrical arrangement of the optical system to collect fluorescent light. In the present BA100 machine, flare appears along the direction of beam scan so that flare is always perpendicular to the transfer direction of the IP. In Fig. 13, we show three oscillation photographs taken around the 111 Bragg point of an $\mathrm{Si}(111)$ wafer with exactly the same conditions. However, the transfer directions in developing the intensities stored in the IP are different to one another. The transfer direction in Fig. 13(a) is from right to left and the flare is seen at the high-angle side from the Bragg point. In Fig. 13(b), the transfer di- rection is from left to right and we see the flare at the lowangle side from the Bragg point. In Fig. 13(c), it is from top to bottom and the flare is seen on the upper side of the Bragg point. We see from these figures that the flare depends on the transfer direction. It always appears before the laser beam scans a strong Bragg point.
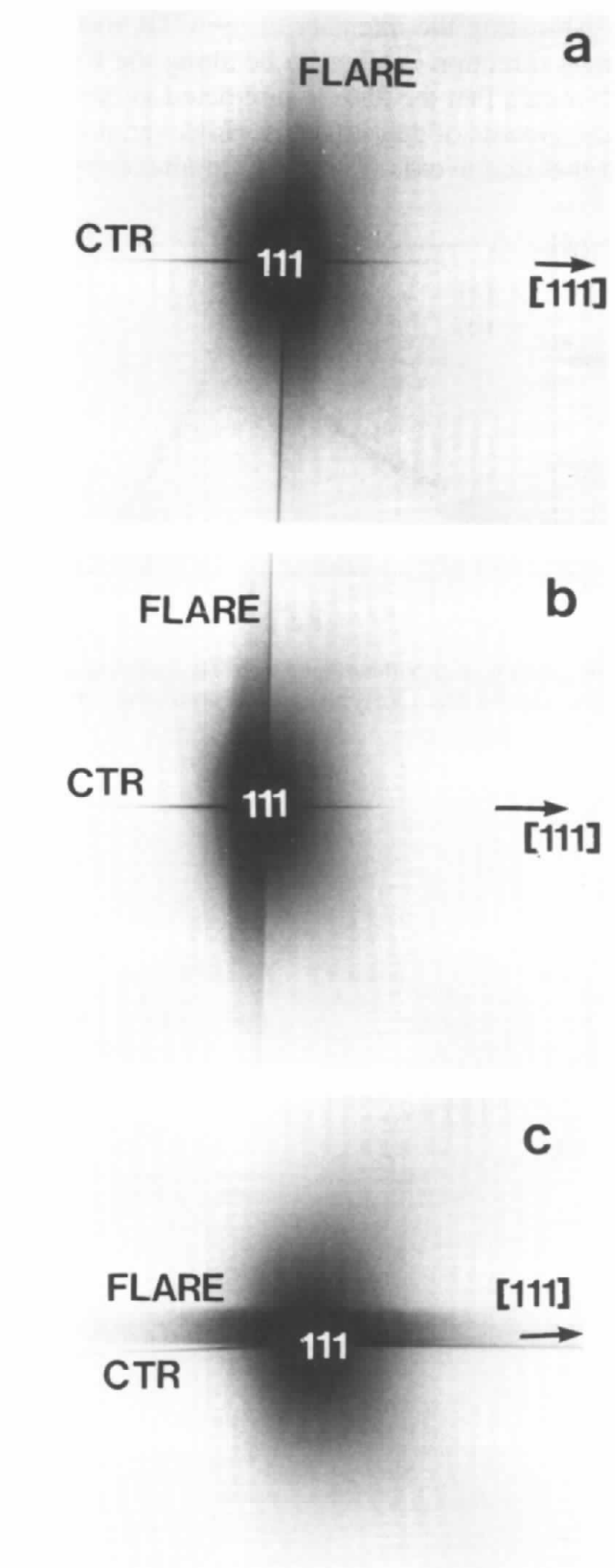

Fig. 13. Three oscillation photographs taken around the 111 Bragg point of an $\mathrm{Si}(111)$ wafer with exactly the same conditions. The transfer directions in developing the intensities stored in the IP are different to one another. The transfer direction is from right to left for $(a)$, from left to right for $(b)$ and from top to bottom for $(c)$. 
In order to see the influence of flare on the profile of the CTR scattering, we estimated the integrated intensity of the CTR scattering from two data sets, Figs. 13(a) and (b), and compare them in Fig. 14. The agreement between them is excellent although the strong part of the flare is on the opposite side of the Bragg point for Figs. 13(a) and $(b)$. This confirms that there is no influence from the flare in estimating the intensity of the CTR scattering if the transfer direction is taken to be along the CTR scattering. It means that the flare is subtracted as background during the process of data analysis. However, it is recommended that one avoids observing simultaneously weak

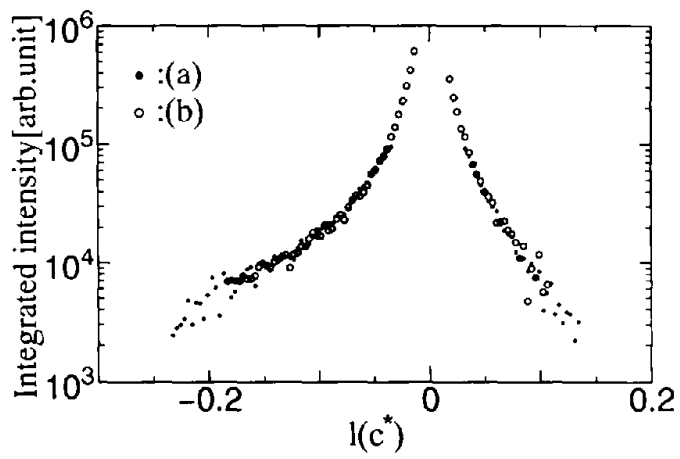

Fig. 14. The intensity distributions along the CTR scattering in Fig. 13; solid circles for Fig. 13(a) and open circles for Fig. 13(b). scattering with strong scattering, such as a Bragg reflection.

\section{References}

amemiya, Y., Matsushita, T., Nakagawa, A., Satow, Y., MiyaHARA, J. \& ChIKAWA, J. (1988). Nucl. Instrum. Methods, A266, 645-653.

Andrews, S. R. \& Cowley, R. A. (1985). J. Phys. C, 18, 6427-6439. Barnea, Z., Clapp, R., Creagh, D. C., Sabine, T. M., Stevenson, A. W., White, J. W., Wilkins, S. W., Harada, J., Hashizume, H., Kashihara, Y., Sakata, M., Ohsumi, K. \& ZemB, T. (1989). Rev. Sci. Instrum. 60(7), 2537-2540.

Harada, J. \& Kashiwagura, N. (1989). J. Phys. (Marseille) Colloq. 50, C7-129.

Kashimara, Y., Ixeda, K. \& Harada, J. (1989). Jpn. J. Appl. Phys. 28(10), 2044.

Kashimara, Y., Kawamura, K., Kashiwagura, N. \& Harada, J. (1987). Jpn. J. Appl. Phys. 26(6), L 1029

Kashitara, Y., Kimura, S. \& Harada, J. (1989). Surf. Sci. 214, 477-492.

Kashiwagura, N., Kashihara, Y., Sakata, M., Harada, J., Wilkins, S. W. \& Stevenson, A. W. (1987). Jpn. J. Appl. Phys. 26(12), L2026-L2029.

MiYahara, J., Takahashi, K., AMEmiYa, Y., KamiYa, N. \& SATOW, Y. (1986). Nucl. Instrum. Methods, A246, 572-578.

Robinson, I. K. (1986). Phys. Rev. B, 33(6), 3830-3836.

SAKABE, N. (1991). Nucl. Instrum. Methods, A303, 448-463.

SHIMURA, T., HaRADA, J. \& KaWAMURA, K. (1993). In preparation. Stevenson, A. W., Wilkins, S. W., Harada, J., Kashtwagura, N., OSHIMA, K. \& SAKATA, M. (1988). Acta Cryst. A44, 828-833.

WoOster, W. A. (1962). Diffuse X-ray Reflections from Crystals, chs. 2 and 3. Oxford: Clarendon Press. 